\title{
CPD QUESTIONS
}

Journal 43

Two CPD points are awarded for the correct completion and submission of the questions below.

CPD questionnaires must be completed online via www.cpdjournals.co.za.

After submission you can check the answers and print your certificate.

Questions may be answered up to 6 months after publication of each issue.

This programme is available free of charge to members of the HIV Clinicians Society and SAMA only.

\author{
Regarding chronic genital ulcer disease in HIV-infected \\ individuals \\ 1. True (A) or false (B) - click on the correct answer: \\ Chronic genital ulcer disease in HIV-infected individuals is only observed at \\ low CD4 cell counts and resolves after aetiological treatment with the use \\ of antiretroviral therapy. \\ 2. True (A) or false (B) - click on the correct answer: \\ MRSA superinfection is a common complication of chronic genital ulcer \\ disease in HIV-infected men.
}

\section{Regarding the use of efavirenz during pregnancy}

3. Click on the correct answer:

The current labelling of efavirenz in pregnancy is:

A: Controlled studies show no risk

B: No evidence of risk in humans

C: Risk in pregnancy can not be ruled out

$D$ : Evidence of risk in pregnancy

$X$ : Contra-indicated in pregnancy

4. True (A) or false (B) - click on the correct answer:

There is substantial evidence from clinical research that EFV is associated with birth defects in humans.

5. True (A) or false (B) - click on the correct answer:

Data from the Antiretroviral Pregnancy Register demonstrate that efavirenz is associated with a higher rate of birth defects than both AZT and 3TC.

6. True (A) or false (B) - click on the correct answer:

Data show that among NNRTIs, efavirenz is associated with equal or greater levels of long-term viral suppression than nevirapine.

7. True (A) or false (B) - click on the correct answer:

Women with high CD4 cell counts using nevirapine are at an increased risk of hepatotoxicities, and therefore nevirapine may not be an ideal choice of NNRTI for a strategy for universal ART use in pregnancy (e.g. 'Test and Treat').

8. True (A) or false (B) - click on the correct answer:

Nevirapine is more expensive than efavirenz.

\section{Regarding the coverage of antiretroviral therapy across South} Africa

9. True (A) or false (B) - click on the correct answer:

Calculating the number of patients on ART in South Africa is a simple addition because of consistently high quality health statistics reported uniformly by all ART services.
10. True (A) or false (B) - click on the correct answer: By the middle of 2011, more than 1.5 million South Africans had been started on ART.

11. True (A) or false (B) - click on the correct answer: The proportion of ART-eligible men who are receiving ART is higher than the proportion of eligible women receiving ART.

12. True (A) or false (B) - click on the correct answer: The numbers of individuals starting ART nationwide fell far short of the targets set by the 2007-2011 National Strategic Plan.

\section{Regarding socioeconomic v. cultural explanations for the spread} of HIV in South Africa

13. True (A) or false (B) - click on the correct answer:

Concurrent partnerships are likely to play a role in understanding the spread of HIV infection at a population level.

Regarding the impact of breastfeeding by HIV-infected mothers on the health of exposed infants

14. True (A) or false (B) - click on the correct answer: Antiretroviral drug interventions such as nevirapine prophylaxis for infants, and/or maternal use of multi-drug ARV regimens, can be used during breastfeeding to reduce the risk of transmission.

15. At a population level, the benefits of formula feeding (related to prevention of HIV infection) are likely to outweigh the risks (in other sources of morbidity and mortality) only in countries where the infant mortality rate is less than 10 per 1000 .

\section{Regarding the use of stavudine}

16. True (A) or false (B) - click on the correct answer:

Both stavudine and tenofovir can lead to thymidine analogue mutations (TAMs).

17. Although lipo-atrophy occurs with stavudine, it is highly reversible after stopping stavudine use.

18. Neuropathy is a complication of stavudine use that may be more likely in patients receiving $\mathrm{TB}$ co-treatment.

19. Most toxicities associated with stavudine use take place in the first year on therapy.

20. Most toxicities associated with stavudine use appear to be dose-dependent. 